International Mathematical Forum, Vol. 8, 2013, no. 5, 223 - 228

\title{
Conjugate Loops: An Introduction
}

\author{
A. Batool, A. Shaheen and A. Ali \\ Department of Mathematics \\ Quaid-i-Azam University, Islamabad, Pakistan \\ afshan_batoolqau@yahoo.com \\ saqaas12@gmail.com \\ dr_asif_ali@hotmail.com
}

\begin{abstract}
In general conjugate of an element does not have any sense in loops. We introduce a new class of loops that possess conjugates of their elements. We call such loops conjugate loops. Conjugate loops are loops in which the identity $x\left(y x^{-1}\right)=(x y) x^{-1}$ holds. We prove that such loops satisfy (1) An IP loop is conjugate $\Longleftrightarrow$ it is flexible, (2) Conjugacy is not an equivalence relation in conjugate loops. We also prove several other related results for conjugate loops
\end{abstract}

\section{Mathematics Subject Classification: 20N99}

Keywords: conjugate loop, flexible IP loop, conjugate IP loop.

\section{Introduction}

A conjugate loop is a set $L$ and a binary operation "." where $L$ contains an identity $e$ such that $x \cdot e=x=e \cdot x \forall x \in L$, and where each $x \in L$ has a twosided inverse $x^{-1}$ such that for all $y \in L, L_{x} R_{x^{-1}}=R_{x^{-1}} L_{x}$ or equivalently,

$$
x \cdot\left(y \cdot x^{-1}\right)=(x \cdot y) \cdot x^{-1} .
$$

Following Bruck [4], a loop $L$ is said to be diassociative if the subloop $\langle x, y\rangle$ generated by any two elements is group. Obviously, every diassociative loop is a conjugate loop. Moufang loop is defined to be a loop satisfying $(x(y z)) x=$ $(x y)(z x)$. A loop is said to have the inverse property, and is called an IP loop, $\Longleftrightarrow x^{-1}(x y)=e=(y x) x^{-1}$. Moufang loops are always IP loops, and flexible loops (a loop satisfying $x(y x)=(x y) x$ ), see [6]. Steiner loop, which may be defined to be an IP loop of exponent two (follows from Lemma 2.2, of [10]), is obviously a diassociative; in fact each $\langle x, y\rangle$ is a boolean group (of order 1, 2, or 4). Less obviously, by Moufang Theorem, every Moufang loop is a 
diassociative loop. C-loops, introduced by Fenyves [5], are loops satisfying the equation $x(y(y z))=((x y) y) z$. These are IP [5, Theorem 4] and are Steiner [10, Lemma 2.1]. C-loop which is not Moufang, is just the 10-element Steiner loop, Table 1 of [5]. Flexible C loops are diassociative [10, Lemma 4.4].

Now in Theorem 1.1, we will show that conjugate IP loops are actually flexible IP loops.

Theorem 1.1. Let $L$ be an IP loop then $L$ is a conjugate $\Longleftrightarrow$ it is flexible.

Proof. Suppose $L$ is a conjugate loop, we have to show that it is flexible

$$
\begin{aligned}
(x(y x)) x^{-1} & =x\left((y x) x^{-1}\right) & & \text { since } L \text { is a conjugate loop } \\
& =x y & & \text { by inverse property } \\
\left(x(y x) \cdot x^{-1}\right) x & =(x y) x & & \text { post multiplying by } x \\
x(y x) & =(x y) x & & \text { by inverse property. }
\end{aligned}
$$

So $L$ is flexible loop. Now, conversely suppose that $L$ is flexible loop

$$
\begin{aligned}
\left(\left(x\left(y x^{-1}\right)\right) x\right. & =x\left(\left(y x^{-1}\right) x\right) & & \text { by flexibility } \\
& =x y & & \text { by inverse property } \\
\left(x\left(y x^{-1}\right) \cdot x\right) x^{-1} & =(x y) x^{-1} & & \text { post multiplying by } x^{-1} \\
x\left(y x^{-1}\right) & =(x y) x^{-1} . & &
\end{aligned}
$$

\subsection{Smallest conjugate loop}

The smallest nonassociative noncommutative flexible loop is of order 5, and it is unique. Its multiplication table is given in Example 1.2. We can see immediately that this loop has exponent 2. It is therefore a Steiner loop and hence the smallest nonassociative conjugate loop.

Example 1.2. The smallest nonassociative conjugate loop.

$\begin{array}{lllll}1 & 2 & 3 & 4 & 5 \\ 2 & 1 & 4 & 5 & 3 \\ 3 & 5 & 1 & 2 & 4 \\ 4 & 3 & 5 & 1 & 2 \\ 5 & 4 & 2 & 3 & 1\end{array}$

Above loop is nondiassociative loop which does not satisfy inverse property.

Smallest nonassociative IP loop is of order 7 , which is given in [1, p. 56], is also a flexible loop and hence a conjugate loop. Now a nonflexible conjugate loop is given in Example 1.3. 
Example 1.3. Non flexible conjugate loop.

$\begin{array}{llllllll}1 & 2 & 3 & 4 & 5 & 6 & 7 & 8 \\ 2 & 1 & 5 & 6 & 7 & 8 & 4 & 3 \\ 3 & 8 & 6 & 1 & 4 & 2 & 5 & 7 \\ 4 & 7 & 1 & 5 & 2 & 3 & 8 & 6 \\ 5 & 3 & 4 & 7 & 8 & 1 & 6 & 2 \\ 6 & 4 & 8 & 3 & 1 & 7 & 2 & 5 \\ 7 & 5 & 2 & 8 & 6 & 4 & 3 & 1 \\ 8 & 6 & 7 & 2 & 3 & 5 & 1 & 4\end{array}$

Many properties that we take for granted in groups do not hold in conjugate loops. Now we will discuss some properties which conjugate loop fails to satisfy.

\subsection{Conjugacy is not an equivalence relation}

Unlike groups, conjugacy is not an equivalence relation in conjugate loops.

\subsubsection{Reflexive:}

Let $L$ be a conjugate loop. Since

$$
(x x) x^{-1}=x\left(x x^{-1}\right)=x(e)=x \quad \forall x \in L
$$

where $e$ is the identity of $L$. So $L$ is reflexive.

\subsubsection{Symmetric:}

Following loop shows that symmetric property does not hold in a conjugate loop.

$\begin{array}{llllllll}1 & 2 & 3 & 4 & 5 & 6 & 7 & 8 \\ 2 & 1 & 4 & 3 & 6 & 7 & 8 & 5 \\ 3 & 4 & 2 & 1 & 7 & 8 & 5 & 6 \\ 4 & 3 & 1 & 2 & 8 & 5 & 6 & 7 \\ 5 & 6 & 7 & 8 & 4 & 1 & 2 & 3 \\ 6 & 7 & 8 & 5 & 1 & 2 & 3 & 4 \\ 7 & 8 & 5 & 6 & 2 & 3 & 4 & 1 \\ 8 & 5 & 6 & 7 & 3 & 4 & 1 & 2\end{array}$

In this loop 5 is the conjugate of 8 , because there exists $3 \in L$ such that $3\left(8.3^{-1}\right)=5$. But we cannot find any $g \in L$ such that $g\left(5 \cdot g^{-1}\right)=8$. So, 8 is not a conjugate of 5 , hence symmetric property does not hold in above loop. 


\subsubsection{Transitive:}

Above loop also shows that transitive property does not hold in conjugate loops. Here 8 is the conjugate of 6 and 6 is the conjugate of 5 but clearly 8 is not a conjugate of 5 . Hence conjugacy is not an equivalence relation in conjugate loops.

\subsection{Conjugate of a subloop}

Let $L$ be a conjugate loop, $x \in L$ and $H \leq L$. Then the conjugate of $H$ with respect to $x$ is,

$$
x^{-1} H x=\left\{x^{-1} h x: \forall h \in H\right\} .
$$

\subsubsection{Conjugate of a subloop is not a subloop}

We know that in groups, conjugate of a subgroup is again a subgroup. But following example shows that it is not true in the case of conjugate loops.

Example 1.4. Consider the following conjugate loop of order 8.

$\begin{array}{llllllll}1 & 2 & 3 & 4 & 5 & 6 & 7 & 8 \\ 2 & 1 & 4 & 3 & 7 & 8 & 5 & 6 \\ 3 & 4 & 2 & 1 & 6 & 5 & 8 & 7 \\ 4 & 3 & 1 & 2 & 8 & 7 & 6 & 5 \\ 5 & 8 & 6 & 7 & 3 & 1 & 2 & 4 \\ 6 & 7 & 5 & 8 & 1 & 3 & 4 & 2 \\ 7 & 6 & 8 & 5 & 4 & 2 & 3 & 1 \\ 8 & 5 & 7 & 6 & 2 & 4 & 1 & 3\end{array}$

Here consider the subloop $H=\{1,2\}$, then $5 H 5^{-1}=\{1,4\}$ which is not a subloop of the above loop.

Theorem 1.5. In a conjugate loop $L$, if $H \unlhd L$ then, $\forall x \in L, H^{x}=H$.

Proof. Let $L$ be a conjugate loop and $H \unlhd L$, then by the definition of normal subloop

$$
\begin{aligned}
H^{x} & =x\left(H x^{-1}\right)=x\left(x^{-1} H\right) \quad \forall x \in L \\
& =\left(x x^{-1}\right) H \quad \forall x \in L \\
& =H .
\end{aligned}
$$




\subsection{Nucleus}

The nucleus $N$ of a loop $L$ is always a subgroup of $L$, but it is not necessarily a normal subgroup of $L$. Even when $L$ is a conjugate loop, its nucleus does not have to be normal in L. (See Example 1.6).

Example 1.6. Conjugate loop with nucleus that is not normal.

$\begin{array}{llllllll}1 & 2 & 3 & 4 & 5 & 6 & 7 & 8 \\ 2 & 1 & 5 & 6 & 3 & 4 & 8 & 7 \\ 3 & 6 & 1 & 7 & 8 & 2 & 4 & 5 \\ 4 & 5 & 7 & 1 & 2 & 8 & 3 & 6 \\ 5 & 4 & 2 & 8 & 7 & 1 & 6 & 3 \\ 6 & 3 & 8 & 2 & 1 & 7 & 5 & 4 \\ 7 & 8 & 4 & 3 & 6 & 5 & 2 & 1 \\ 8 & 7 & 6 & 5 & 4 & 3 & 1 & 2\end{array}$

Check that 2 is in the nucleus and $3^{-1} \cdot 2.3=8$. But 8 is not in the nucleus, since.5.(4.8) $\neq(5.4)$.8.

\subsection{Lagrange-like property}

A finite loop $L$ is said to have the weak Lagrange property if the order of any subloop of $L$ divides the order of $L$.

The order of any subloop of a finite Moufang loop $M$ divide the order of $M[7$, Lagrange's theorem]. But Steiner loops (and hence conjugate loops) do not have the weak Lagrange property. This is illustrated by the loop $L$ with multiplication table given in Example 1.2 which possesses a subloop $\{1,2\}$ of order 2.

\subsection{Cauchy-like property}

A finite 3-power associative loop is said to have the weak Cauchy property if for any prime $p$ dividing the order of the loop there is an element of order $p$.

Since there are Steiner loops of order different from $2^{k}$, yet all Steiner loops have exponent 2, it is clear that Steiner loops (and thus conjugate loops) do not have the weak Cauchy property. Example 1.2 illustrates this nicely (and minimally) for $p=5$.

Acknowledgement: Thanks to Muhammad Shah, Quaid-e-Azam University Islamabad Pakistan, for many useful comments and suggestions. 


\section{References}

[1] A. Ali and J. Slaney. Generating Loops with the Inverse Property, Proc. ESARAM, 55(2008), 55-66.

[2] R. Artzy, Relations between loops identities, Proc. Amer. Math. Soc. , Vol. 11, 6 (1960), 847-851.

[3] R. H. Bruck, Pseudo-Automorphism and Moufang loops, Proc. Amer. Math. Soc. 2(1951), 66-71.

[4] R. H.Bruck, A Survey of Binary Systems, third printing, corrected, Springer- Verlag, 1971.

[5] F. Fenyves, Extra loop II. On loops with identities of Bol-Moufang type, Publ. Math. Debrecen, 16 (1969), 187-192.

[6] E. G. Goodaire, Eric Jespers and Cesar Polcino Miles , Alternative loop ring, North Holland Math., 1996.

[7] A. N.Grishkov, A. V. Zavarnitsine, Langrange's theorem for Moufang loops, Math. Proc. Camb. Phil. Soc. (2004), 101-116.

[8] M. K. Kinyon, K. Kunen and J. D. Phillips, A generalization of Moufang and Steiner loops, Algebra Universalis, 48 (2002), 81-101.

[9] H. O. Pflugfelder, Quasigroups and Loops: Introduction, Sigma Series in Pure Mathematics 7, Heldermann Verlag Berlin, (1990).

[10] J. D. Philips, P. Vojtechovsky, C-loops: An Introduction, Pupl. Math. Debrecen, 68 (2006), 1-2, 115-137.

\section{Received: October, 2012}

